\title{
The Deterministic Circuit Model for Noise Influence on the Averaged Transient Responses of Large-scale Nonlinear ICs Analyzed with Itô's Stochastic Differential Equations
}

\author{
MAGNUS WILLANDER*, YEVGENY MAMONTOV ${ }^{\dagger}$ and JONATHAN VINCENT ${ }^{\ddagger}$ \\ Laboratory of Physical Electronics and Photonics, MC2, School of Physics and Engineering Physics, \\ Chalmers University of Technology and Göteborg University, SE-412 96 Göteborg, Sweden
}

\begin{abstract}
The second-order nonrandom ordinary differential equation (ODE) system derived as the noise-source-aware model for expectations of solutions of Itô's stochastic differential equation (ISDE) system is discussed in connection with large-scale integrated circuits (ICs). The work explains the reason why the new model consistently allows for the noise-induced phenomena in the expectations, namely, stochastic resonance, stochastic linearization, stochastic self-oscillations and stochastic chaos. The case of stochastic resonance is considered as an example. In spite of the fact that the above second-order model is more complex than the nonrandom first-order IC ODE system for the expectations commonly used in engineering, an efficient practical technique for its implementation is proposed. The corresponding predicted computing time is only in 2.5 times greater than in the case of the first-order model which does not include any noise-source influence upon the expectations of the modelled IC responses.
\end{abstract}

Keywords: Noise in nonlinear integrated circuits; Circuit equation system; Itô's stochastic differential equation system; Noise-induced phenomena in expectation; Stochastic resonance; The second-order ordinary differential equation system for expectation

\section{INTRODUCTION}

The common integrated-circuit (IC) equation system is ordinary differential equation (ODE) system

$$
d x / d t=g(t, x)
$$

with initial condition

$$
\left.x\right|_{t=t_{o}}=e_{o}
$$

where $t \in \mathbb{R}$ is the time, $\mathbb{R}=(-\infty, \infty), x \in \mathbb{R}^{d}$ $(d \geq 1)$ is the vector of the circuit variables

*Tel.: +46 31772 2093, e-mail: mwi@fy.chalmers.se

${ }^{\dagger}$ Corresponding author. Tel.: +46 31772 3489, Fax: +46 31772 2092, e-mail: yem@fy.chalmers.se

${ }^{\ddagger}$ Tel.: +46 31772 5478, e-mail: vincent@,fy.chalmers.se 
(voltages, currents), $g$ is a sufficiently smooth real $d$-vector-function defined for all $(t, x) \in \mathbb{R}^{d+1}$, $t_{\mathrm{o}} \in \mathbb{R}$ is the initial time point and $e_{\mathrm{o}} \in \mathbb{R}^{d}$ is the value of vector $x$ at moment $t_{\mathrm{o}}$. Solution $x=e(t)$ of the initial-value problem (1.1), (1.2) at $t \geq t_{\mathrm{o}}$ describes the circuit behavior. System (1) is, as a rule, large-scale (or high-dimensional) in the sense that

number $d$ is much greater than a few units.

In many cases, number $d$ is on the order of a few tens or hundreds (or greater).

Noise in ICs is usually (e.g. [1-6]) described by means of Itô's stochastic differential equation (ISDE) system (e.g. [7, 8])

$$
d x=g(t, x) d t+h(t, x) d W(\xi, t)
$$

with initial condition

$$
\left.x\right|_{t=t_{\mathrm{o}}}=\chi_{\mathrm{o}}(\xi)
$$

where $h(t, x)$ is a sufficiently smooth real $d \times d$ matrix-function defined for all $(t, x) \in \mathbb{R}^{d+1}$, $W(\xi, t)$ is a $d$-dimensional Wiener stochastic process, $\xi$ is elementary event and function $\chi_{\mathrm{o}}$ is the initial random variable with probability density $\rho_{\mathrm{o}}$. The initial-value problem (1.4), (1.5) presents the stochastic generaliztion of deterministic problem (1.1), (1.2). Solution $x=\chi(\xi, t)$ of problem (1.4), (1.5) at $t \geq t_{\mathrm{o}}$ is a stochastic process which is also a diffusion process (e.g. [8, (9.3.1)]). It describes the stochastic behavior of the modelled IC. In so doing, vector $g(t, x)$ and matrix

$$
H(t, x)=h(t, x)[h(t, x)]^{\mathrm{T}}, \quad \text { for all }(t, x) \in \mathbb{R}^{d+1},
$$

are called the drift vector and the diffusion matrix of solutions of ISDE (1.4).

The key deterministic (i.e., nonrandom) characteristic of process $\chi$ is its expectation $E[\chi(\cdot, t)]$. It is analogous to the above solution $e(t)$ of deterministic problem (1.1), (1.2) so, in the stochastic case, function $e$ can be interpreted as follows

$$
e(t)=E[\chi(\cdot, t)], \quad \text { for all } t \geq t_{\mathrm{o}} .
$$

In so doing, initial value $e_{\mathrm{o}}$ for expectation (1.7) is determined from (1.5), i.e.,

$$
e\left(t_{\mathrm{o}}\right)=e_{\mathrm{o}}=\int_{\mathbb{R}^{d}} x_{\mathrm{o}} \rho_{\mathrm{o}}\left(x_{\mathrm{o}}\right) d x_{\mathrm{o}} .
$$

where $x_{\mathrm{o}}$ denotes values of initial random variable $\chi_{\mathrm{o}}$.

Modelling noise in ICs (both analogue and digital) based on the stochastic initial-value problem (1.4), (1.5) is a branch of electroniccircuit theory. The details of the formulation of functions $g$ and $h$ in (1.4) coming from the circuit configuration and semiconductor-device parameters can be found, for example, in [1-6] and the references therein. These papers do not constitute a comprehensive list of the literature on the topic. They do however include most of the key points of the corresponding treatment. The works [1-6] also discuss the physical interpretation of ISDE system (1.4). In so doing, the first term on the right-hand side of (1.4) is regarded as the noiseless version of the circuit model ( $c f .(1.1))$ whereas the second one presents the stochastic noise source. The derivation of function $h$ related to this source from the basic physical parameters is developed in $[1,4,5]$. The time-derivative $d W(\xi, t) /$ $d t$ is meaningful only as a generalized stochastic process and, in this sense, is white noise widely used in engineering. Examples of the results of various techniques for numerical solving initialvalue problem (1.4), (1.5) are reported in $[2,3,6]$. The present work does not discuss the above welldocumented theory of stochastic-noise-aware IC model (1.4), (1.5). The work deals with a problem which concerns ISDE system (1.4) and is described below.

Expectation (1.7) can be regarded as the deterministic response of the circuit and plays a 
crucial role in engineering analysis and design. If ISDE system (1.4) is linear in $x$, then, as is wellknown (e.g. [8, Section 8.2]), expectation $e(t)$ is independent of $h$ and hence of the stochastic noise source. This fact sometimes leads to the simplistic idea (e.g. [3]) that, even if ISDE system (1.4) is nonlinear in $x$, then function $e$ still remains independent of the noise source and, thus, can be determined from familiar deterministic initialvalue problem (1.1), (1.2).

However, this is not true in general as shown by many results in natural sciences and engineering (e.g. [9-13]) (in particular, in semiconductor systems [14-19]) associated with the noise-induced phenomena in expectation $e(t)$ : stochastic resonance, stochastic linearization, stochastic selfoscillations, stochastic phase transistions, stochastic chaos. These effects represent the influence of noise upon function $e$. This naturally poses the questions: is it possible to derive an ODE system (similar to (1.1)) for $e$ in the stochastic case (1.4), (1.5) and, if yes, to develop an efficient practical technique for the derived system? This is the problem which the present works is devoted to. The work applies the previous results of the authors and focuses on the corresponding practical issues.

\section{THE SECOND-ORDER ODE SYSTEM FOR THE EXPECTATION}

The above mentioned drawback of the model (1.1), (1.2) for expectation $e$, i.e., $e$ is independent of function $h$ in (1.4), is removed if $e$ is determined as the solution of the second-order ODE system

$$
d^{2} x / d t^{2}=\partial g(t, x) / \partial t+[\partial g(t, x) / \partial x] g(t, x)+\bar{h}(t, x)
$$

with initial conditions (1.2) and

$$
d x /\left.d t\right|_{t=t_{\mathrm{o}}}=g\left(t_{\mathrm{o}}, e_{\mathrm{o}}\right)
$$

where

$$
\begin{gathered}
\bar{h}(t, x)=\left(\bar{h}_{1}(t, x), \bar{h}_{2}(t, x), \ldots, \bar{h}_{d}(t, x)\right)^{T}, \\
\text { for all }(t, x) \in \mathbb{R}^{d+1}, \\
\bar{h}_{k}(t, x)=(1 / 2) \operatorname{tr}\left[H(t, x) \partial^{2} g_{k}(t, x) / \partial x^{2}\right], \\
k=1,2, \ldots, d, \quad \text { for all }(t, x) \in \mathbb{R}^{d+1},
\end{gathered}
$$

$g_{k}$ is the $k$ th entry of vector $g, \operatorname{tr}(\cdot)$ is the trace of matrix (i.e., the sum of the entries of its principal diagonal) and $e_{\mathrm{o}}$ is described with (1.8). Condition (2.2) follows from (1.1) and (1.2). The description (2.1), (1.2), (2.2) for expectation $e$ was rigorously derived under rather mild assumptions in [20, Theorem 4 and Section 4.2] (see also [21, Theorem 2.4 and Section 2.3.2]).

The initial-value problems (1.1), (1.2) and (2.1), (1.2), (2.2) are equivalent if and only if $\bar{h}(t, x) \equiv 0$. If this relation does not hold, then the latter problem can not be obtained from the former one. Thus, vector $\bar{h}(t, x)$ (see (2.3), (2.4)) in (2.1) is the new term provided by the second-order ODE (2.1).

The system (2.1) is of the second order, but it is also possible to derive analogous ODE systems of the higher orders. This topic was developed in [20, Section 4.2] (see also [21, Section 2.3.3]). Note, the higher the order of the system is, the more accurate the description of the expectation is and the more refined the way the noise is allowed for becomes. Complexity of the higher order models, however, rapidly increases with increase in the order. In spite of that, these models can be required in applied problems.

\section{MODEL FOR NOISE-INDUCED PHENOMENA IN THE EXPECTATION: CASE OF STOCHASTIC RESONANCE}

The advances of the last decade demonstrate the keen interest of many researchers and engineers in 
various noise-induced phenomena in expectations of stochastic processes: stochastic resonance, stochastic linearization, stochastic self-oscillations, stochastic phase transitions, stochastic chaos. The first part of these terms, the word "stochastic", points out that the listed effects are due to the influence of noise upon the expectations. There is a vast literature on the topic (e.g. [9-13] and the references therein).

Stochastic resonance, linearization, self-oscillations, phase transitions, chaos are related to different aspects of stochastic systems. However, they all have something in common. Namely, they are associated with the noise and the nonlinearities of the system. These are the features which are taken into account with expression (2.4) for vector (2.3) in ODE (2.1). Indeed, the nonlinearities in (2.4) are presented by the second derivatives of the entries of drift function $g$, whereas the noise is accounted with diffusion function $H$ (see (1.6)). As an example, we consider the connection of ODE (2.1) with only stochastic resonance.

Stochastic resonance is usually associated with the following features.

- The stochastic system under consideration is nonlinear. (No stochastic resonance in linear stochastic systems has been found so far.)

- The system is driven by a deterministic periodic input signal.

- The nonlinearities and the periodic input cause stochastic resonance in the periodic output signal.

- Stochastic resonance in the output results in (e.g. [12, Section 4.1]) an increase in the expectation of the periodic output signal or even the stronger phenomenon that the signalto-noise ratio (SNR) of the periodic output signal achieves a local maximum at a nonzero standard deviation (or root-mean-square value) of the signal.

The improvement in the output-signal amplitude or in the SNR is the most important practical advantage of stochastic resonance.
According to the above features, we shall consider the case when

$$
\begin{aligned}
& g(t, x)=g(v, x), \quad H(t, x)=H(v, x) \\
& v=\bar{v}+\boldsymbol{v}(t)
\end{aligned}
$$

where $\bar{v}$ is a vector independent of $(t, x)$ and $\boldsymbol{v}(t)$ is a small periodic signal. In so doing, we analyze the periodic solution of system (2.1) under condition (3.1), i.e.

$$
\begin{aligned}
\frac{d^{2} x}{d t^{2}}= & \frac{\partial g(\bar{v}+\mathbf{v}(t), x)}{\partial v} \frac{d \boldsymbol{v}(t)}{d t} \\
& +\frac{\partial g(\bar{v}+\mathbf{v}(t), x)}{\partial x} g(\bar{v}+\mathbf{v}(t), x) \\
& +\bar{h}(\bar{v}+\mathbf{v}(t), x)
\end{aligned}
$$

The periodic solutions can be studied by means of the well-known techniques. The corresponding theory can be found, for example, in [22-26]. More practice-oriented analytical treatments are also available (like the finite-equation method by the authors [27, 28]). However, the general theory does not always explain the resonance in a compact form. To fill this gap, we simplify the ODE (3.2).

We assume that:

- signal $\boldsymbol{v}(t)$ is small enough to enable one to replace ODE (3.2) with its small-signal representation

$$
\begin{aligned}
d^{2} x / d t^{2}= & {[\partial g(\bar{v}, x) / \partial v] d \mathbf{v}(t) / d t } \\
& +[\partial g(\bar{v}, x) / \partial x] g(\bar{v}, x)+\bar{h}(\bar{v}, x)
\end{aligned}
$$

- the autonomous version

$$
d^{2} x / d t^{2}=[\partial g(\bar{v}, x) / \partial x] g(\bar{v}, x)+\bar{h}(\bar{v}, x)
$$

of the system (3.3) has the unique equilibrium point $x=\bar{x}$ such that $\operatorname{det}[\partial g(\bar{x}) / \partial x] \neq 0$, number $\left\|[\partial g(\bar{x}) / \partial x]^{-1} \bar{h}(\bar{x})\right\|$ is much less than the typical values of $\|\bar{g}(x)\|$ for all $x \approx \bar{x}$ and

$$
\operatorname{det}\left\{\lambda^{2} I-[\partial g(\bar{v}, \bar{x}) / \partial x] \lambda-[\partial \bar{h}(\bar{v}, \bar{x}) / \partial x]\right\}=0 \Rightarrow \operatorname{Re} \lambda<0
$$

where $I$ is the $d \times d$ identity matrix. 
Then the ODE system (3.4) can be approximated with the system

$$
\begin{gathered}
d^{2}(x-\bar{x}) / d t^{2}=[\partial g(\bar{v}, \bar{x}) / \partial x] d(x-\bar{x}) / d t \\
+[\partial \bar{h}(\bar{v}, \bar{x}) / \partial x](x-\bar{x}) \\
\text { for all } x \approx \bar{x}
\end{gathered}
$$

so the corresponding version of (3.3) is the asymptotically stable linear ODE

$$
\begin{aligned}
\frac{d^{2}(x-\bar{x})}{d t^{2}}= & \frac{\partial g(\bar{v}, \bar{x})}{\partial v} \frac{d \boldsymbol{v}(t)}{d t} \\
& +\frac{\partial g(\bar{v}, \bar{x})}{\partial x} \frac{d(x-\bar{x})}{d t} \\
& +\frac{\partial \bar{h}(\bar{v}, \bar{x})}{\partial x}(x-\bar{x}), \quad \text { for all } x \approx \bar{x} .
\end{aligned}
$$

As is well-known, if signal $\boldsymbol{v}(t)$ is a harmonic, then the linear ODE (3.5) has the unique periodic solution which is also a harmonic with the frequency identical to that of signal $\boldsymbol{v}(t)$. A fairly common derivation which is mainly of a technical nature shows that the last term on the right-hand side of (3.5), i.e., the term related to function $\bar{h}$, generally causes the resonance in the periodic solution $x-\bar{x}$ even in the one-dimensional case. The expressions (2.3), (2.4) for $\bar{h}$ stress that this resonance is due to both the nonlinearity of drift function $g$ and the randomness-related diffusion function $H$ (see (1.6), (1.4)). Such resonance is known as stochastic as is mentioned at the beginning of this section.

Stochastic linearization, self-oscillations, phase transitions and chaos can also be analyzed on the basis of the corresponding treatments of the second-order ODE system (2.1). In so doing, the effects associated with vector $\bar{h}(t, x)$ are of a key importance.

The initial-value problem (2.1), (1.2), (2.2) presents a more accurate description of expectation function $e$ than the initial-value problem (1.1), (1.2). The advantage of (2.1) compared to (1.1) is that it by means of (2.3) and (2.4) includes dependence of the solutions on diffusion function $H$. The disadvantage is that (2.1) is a more complex ODE than (1.1). Section 4 considers this problem in detail and suggests the way to eliminate it.

\section{PRACTICALLY EFFICIENT IMPLEMENTATION OF THE SECOND-ORDER ODE SYSTEM}

The initial-value problem (2.1), (1.2), (2.2) can numerically be solved by means of the same techniques as those developed for the initial-value problem (1.1), (1.2), for instance, the semi-explicit Euler method (e.g. [29]). The overall efficiency is proportional to the computing expense required to evaluate the right-hand side of the ODE system once. In case of system (2.1), the expense is mainly due to calculation of matrix $\partial g(t, x) / \partial x$ and vector (2.3) in (2.1). This procedure involves not only $d$ nonlinear scalar functions $g_{1}, g_{2}, \ldots, g_{d}$ but also all their $x$-derivatives of the first and second orders and all entries of symmetric matrix $H(t, x)$. The total number $N$ of the nonlinear scalar functions of $d$ scalar variables is $d\left(d^{2}+4 d+1\right) / 2$ or

$$
N \approx d^{3} / 2, \quad \text { for high } d(\operatorname{see}(1.3)),
$$

which is an unacceptable amount of computations. This problem can be eliminated if one applies the technique below to simultaneously evaluate matrix $\partial g(t, x) / \partial x$ and vector (2.3) in (2.1).

Since diffusion matrix $H(t, x)$ is non-negative definite symmetric (see (1.6)), then there exists square matrix $\tilde{A}(t, x)$ such that

$$
H(t, x)=\tilde{A}(t, x)[\tilde{A}(t, x)]^{\mathrm{T}}, \quad \text { for all }(t, x) \in \mathbb{R}^{d+1} .
$$

We denote the $l$ th column of matrix $\tilde{A}(t, x)$ with $\tilde{a}_{l}(t, x)$, i.e., $\tilde{A}(t, x)=\left(\tilde{a}_{1}(t, x), \tilde{a}_{2}(t, x), \ldots, \tilde{a}_{d}(t, x)\right)$ 
for all $(t, x) \in \mathbb{R}^{d+1}$. Note that matrix $\tilde{A}(t, x)$ satisfying Eq. (4.2) need not to have the same properties as those of solution $h(t, x)$ of (1.6) (e.g. [30], [31, Section 1 in Chapter 6] for the details on the required properties of $h(t, x))$. In particular, matrix $h(t, x)$ need not to be square. This means that solving (4.2) for $\tilde{A}(t, x)$ is a much simpler task than solving (1.6) for $h(t, x)$. As shown below, matrix $\tilde{A}(t, x)$ serves solely to improve the computational efficiency.

One can readily check with the help of (4.2) that the trace in (2.4) can be described with equation

$$
\begin{aligned}
& \operatorname{tr}\left\{H(t, x)\left[\partial^{2} g_{k}(t, x) / \partial x^{2}\right]\right\} \\
& =\sum_{l=1}^{d}\left[\tilde{a}_{l}(t, x)\right]^{\mathrm{T}}\left[\partial^{2} g_{k}(t, x) / \partial x^{2}\right] \tilde{a}_{l}(t, x), \\
& \quad k=1,2, \ldots, d, \quad \text { for all }(t, x) \in \mathbb{R}^{d+1} .
\end{aligned}
$$

The terms summed over in (4.3) are coupled with the values of the functions $g_{k}$ as follows

$$
\begin{aligned}
g_{k}(t, x & \left. \pm c ̧ \tilde{a}_{1}(\mathrm{t}, \mathrm{x})\right) \\
= & g_{k}(t, x) \pm c ̧\left[\partial g_{k}(t, x) / \partial x\right] \tilde{a}_{l}(t, x) \\
& +\left(\varsigma^{2} / 2\right)\left[\tilde{a}_{l}(t, x)\right]^{\mathrm{T}}\left[\partial^{2} g_{k}(t, x) / \partial x^{2}\right] \tilde{a}_{l}(t, x) \\
& \pm \varsigma^{3} C_{k . l}(t, x)+O\left(\varsigma^{4}\right), \\
& k, l=1,2, \ldots, d, \quad \text { for all }(t, x) \in \mathbb{R}^{d+1}
\end{aligned}
$$

where $c ̧$ is a parameter dependent on $t, x, g_{k}$, and $\tilde{a}_{l}$. Quantity $\zeta_{k . l}(t, x)$ depends on functions $g_{k}$ and $\tilde{a}_{l}$ and is independent of $c ̧$. The two last terms on the right-hand side of (4.4) show that parameter $c$ determines how accurate the three first terms on the right-hand side describe the left-hand side. So $c$ should be chosen sufficiently small. It follows from (4.3) that

$$
\begin{aligned}
& {\left[\tilde{a}_{l}(t, x)\right]^{\mathrm{T}}\left[\partial^{2} g_{k}(t, x) / \partial x^{2}\right] \tilde{a}_{l}(t, x)} \\
& \approx c^{-2}\left[g_{k}\left(t, x+c ̧ \tilde{a}_{l}(t, x)\right)-2 g_{k}(t, x)\right. \\
& \left.\quad+g_{k}\left(t, x-c ̧ \tilde{a}_{l}(t, x)\right)\right], \\
& \quad k, l=1,2, \ldots, d, \quad \text { for all }(t, x) \in \mathbb{R}^{d+1},
\end{aligned}
$$

$$
\begin{aligned}
& {[\tilde{A}(t, x)]^{\mathrm{T}}\left[\partial g_{k}(t, x) / \partial x\right]^{\mathrm{T}}} \\
& \quad \approx \frac{1}{2 c}\left(\begin{array}{c}
\left.g_{k}\left(t, x+c ̧ \tilde{a}_{1}(t, x)\right)-g_{k}(t, x)-c ̧ \tilde{a}_{1}(t, x)\right) \\
\left.g_{k}\left(t, x+c ̧ \tilde{a}_{2}(t, x)\right)-g_{k}(t, x)-c ̧ \tilde{a}_{2}(t, x)\right) \\
\vdots \\
\left.g_{k}\left(t, x+c ̧ \tilde{a}_{d}(t, x)\right)-g_{k}(t, x)-c ̧ \tilde{a}_{d}(t, x)\right)
\end{array}\right), \\
& \quad k, l=1,2, \ldots, d, \text { for all }(t, x) \in \mathbb{R}^{d+1},
\end{aligned}
$$

and the error of each of these approximations is $O\left(c^{2}\right)$.

Relation (4.6) presents $d$ systems of linear algebraic equations for $d$ row vectors $\partial g_{1}(t, x) /$ $\partial x, \ldots, \partial g_{d}(t, x) / \partial x$ with the same matrix $[\tilde{A}(t, x)]^{\mathrm{T}}$. Clearly, these systems can be solved uniquely if the matrix is nonsingular. To assure this property, we assume that

$$
\operatorname{det}[H(t, x)] \neq 0, \quad \text { for all }(t, x) \in \mathbb{R}^{d+1},
$$

i.e., non-negative definite symmetric matrix $H(t, x)$ is positive definite. Then Eq. (4.2) can always be solved for nonsingular matrix $\tilde{A}(t, x)$ at any fixed $(t, x) \in \mathbb{R}^{d+1}$. If this procedure is carried out by means of the Choleski method (e.g. [32, §4.6]), then matrix $\tilde{A}(t, x)$ is lower triangular. In this case, solving systems (4.6) with upper triangular matrix $[\tilde{A}(t, x)]^{\mathrm{T}}$ for any $k=1,2, \ldots, d$ is very simple. For improved efficiency, these $d$ systems can be solved in parallel. Note that, at every fixed $(t, x) \in \mathbb{R}^{d+1}$, the Choleski triangular factorization (e.g. [32, (4.2.19), (4.2.20)]) for Eq. (4.2) is carried out only once.

The above considerations show that evaluation of matrix $\partial g(t, x) / \partial x$ and vector (2.3) in (2.1) requires the calculation of only $N=\left(5 d^{2}+d\right) / 2$ nonlinear scalar functions of $d$ scalar variables, i.e.,

$$
N \approx 5 d^{2} / 2, \quad \text { for high } d(\text { see }(1.3))
$$

that is acceptable under condition (1.3). Number $N$ given by (4.8) is in $d / 5$ times less than in case of (4.1). This and feature (1.3) mean that the 
proposed method is efficient and thereby opens a way to practical application of the second-order ODE (2.1). In so doing, entries (2.4) of vector (2.3) are determined with (4.3) and approximate relations (4.5) whereas rows $\partial g_{k}(t, x) / \partial x$ of matrix $\partial g(t, x) / \partial x$ are uniquely evaluated from $d$ systems (4.6) of linear algebraic equations under condition (4.7). Note that value (4.8) of number $N$ is only in 2.5 times greater than that in the case of the firstorder ODE system (1.1).

\section{CONCLUDING REMARKS}

The work briefly discusses the second-order ODE system (2.1) as a model for expectations of the solutions of an IC ISDE system. The ODE system was rigorously derived in the previous works by the authors. The possibility to apply analogous models of the higher orders is also noted.

The key advantage of the second-order ODE system (2.1) compared to the common, first-order ODE system (1.1) (heuristically applied to model the expectations) is that it includes the term $\bar{h}(t, x)$ which takes into account (see (2.3), (2.4)) the influence of the stochastic-noise-source characteristics upon the expectations. This makes the new model suitable to describe various noise-induced phenomena in the expectations, namely, stochastic resonance, linearization, self-oscillations and chaos. As an example, the connection of the model to stochastic resonance is explained in more detail.

In spite of the advanced second-order ODE system (2.1) is more complex than the commonly used first-order one (1.1), the corresponding efficient practical implementation is proposed. The number $N$ of the nonlinear scalar functions of many variables required to be calculated is in the large-scale case (1.3) proportional to the squared number of the equations (or the variables) (see (4.8)) of the system and is only in 2.5 greater than that for the first-order ODE (1.1).

The new model opens a way to consistent and efficient simulation of the noise-induced phenomena in the expectations of solutions of large-scale IC ISDE systems. This modelling and simulation were not available in microelectronics engineering and industry before.

\section{References}

[1] Mamontov, Y. V. and Willander, M., "Thermal noise in silicon bipolar transistors and circuits for low-current operation-Part I: Compact device model", IEICE Trans. Electronics, E78-C, December, 1995.

[2] Casinovi, G. and Ho, R. J., "Computer generation of colored noise for time-domain analysis of integrated circuits", Analog Integrated Circuits and Signal Processing, $11,1996$.

[3] Demir, A., Liu, E. W. Y. and Sangiovanni-Vincentelli, A., "Time-domain non-Monte Carlo noise simulation for nonlinear dynamic circuits with arbitrary excitations", IEEE Trans. CAD ICAS, May, 1996.

[4] Mamontov, Y. V. and Willander, M., "Model for thermal noise in semiconductor bipolar transistors at low-current operation as multi-dimensional diffusion stochastic process", IEICE Trans. Electronics, July, 1997.

[5] Mamontov, Y. V. and Willander, M., "Application of ordinary/partial Itô's stochastic differential equations to modelling noise in semiconductor devices and circuits", Proc. of ProRISC'97, IEEE Benelux Circuits and Systems Chapter, 8th annual Workshop on Circuits, Systems and Signal Processing, November, 1997.

[6] Schein, O. and Denk, G., "Numerical solution of stochastic differential-algebraic equations with applications to transient noise simulation of microelectronic circuit", J. Comput. Appl. Math., 1, 1998.

[7] Gihman, I. I. and Skorohod, A. V. (1972). Stochastic Differential Equations, New York: Springer-Verlag.

[8] Arnold, L. (1974). Stochastic Differential Equations: Theory and Applications, New York: John Wiley \& Sons.

[9] Bulsara, A., Hänggi, P., Marchesoni, F., Moss, F. and Shlesinger, M. (Eds.), Proc. of the NATO Advanced Research Workshop: Stochastic Resonance in Physics and Biology, J. Statist. Phys., January, 1993.

[10] Lin, H. and Yim, S. C. S., "Analysis of a nonlinear system exhibiting chaotic, noisy chaotic, and random chaotic behaviors", J. Appl. Mech., 63, 509, June, 1996.

[11] Gammaitoni, L., Hanggi, P., Jung, P. and Marchesoni, F., "Stochastic resonance", Rev. Modern Phys., January, 1998.

[12] Luchinsky, D. G., McClintock, P. V. E. and Dykman, M. I., "Analogue studies of nonlinear systems", Rep. Prog. Phys., August, 1998.

[13] Millonas, M. (Ed.), Fluctuations and Order: The New Synthesis. New York: Springer-Verlag, 1996.

[14] Bulsara, A. R., Jacobs, E. W. and Schieve, W. C., "Noise effects in a nonlinear dynamic system: The RF superconducting quantum interference device", Phys. Rev. A., 15 October, 1990.

[15] Mantegna, R. N. and Spagnolo, B., "Stochastic resonance in a tunnel diode", Phys. Rev. E, March, 1994.

[16] Iannelli, J. M., Yariv, A., Chen, T. R. and Zhuang, Y. H., "Stochastic resonance in a semiconductor distributed feedback laser", Appl. Phys. Lett., 17 October, 1994. 
[17] Makarov, D. E. and Makri, N., "Stochastic resonance and nonlinear response in double quantum-well structures", Phys. Rev. B, 15 July, 1995.

[18] Godivier, X., Rojas-Varela, J. and Chapeau-Blondeau, F., "Noise-assisted signal transmission via stochastic resonance in a diode nonlinearity", Electron. Letters, September, 1997.

[19] Neff, J. D., Patel, G. N., Meadows, B. K., DeWeerth, S. P. and Ditto, W. L., "Spatiotemporal dynamics of a stochastic VLSI array", Proc. of the 1998 IEEE Int. Sump. on Circuits and Systems, ISCAS'98, Vol. 3, June, 1998.

[20] Mamontov, Y. V., Willander, M. and Lewin, T., "Modelling of high-dimensional diffusion stochastic process with nonlinear coefficients for engineering applications-Part 1: Approximations for expectation and variance of nonstationary process", Math. Models Methods Appl. Sci., November, 1999.

[21] Mamontov, Y. V. and Willander, M. (2001). HighDimensional Nonlinear Diffusion Stochastic Processes. Modelling for Engineering Applications, Singapore: World Scientific, ISBN 981-02-4385-5.

[22] Demidovič, B. P. (1967). Lectures on the Mathematical Theory of Stability, Moscow: Nauka (Mathematical Reviews \# 1716).

[23] Hale, J. K. (1980). Ordinary Differential Equations, New York: Robert E. Krieger Publishing.
[24] Nayfeh, A. H. and Balachandran, B. (1995). Applied Nonlinear Dynamics: Analytical, Computational, and Experimental Methods, New York: John Wiley \& Sons.

[25] Pliss, V. A. (1966). Nonlocal Problems of the Theory of Oscillations, New York - London: Academic Press.

[26] Pontryagin, L. S. (1962). Ordinary Differential Equations, Reading (MA, USA), Addison-Wesley Publishing.

[27] Mamontov, E. V. (1989). "An equilibrium solution of a system of nonautonomous linear partial differential equations", Theoretical and Numerical Studies of Boundary Value Problems (Mathematical Reviews \# 90j:00013; Current Mathematical Publications \# 1044 578).

[28] Mamontov, Y. V. and Willander, M., "Asymptotic method of finite equation for bounded solutions of nonlinear smooth ODEs", Math. Japonica, November, 1997.

[29] Hall, G. and Watt, J. M. (1976). Modern Numerical Methods for Ordinary Differential Equations, Oxford: Clarendon Press.

[30] Freidlin, M. I., "On the factorization of non-negative definite matrices", Theory Probab. Appl., 1968.

[31] Friedman, A. (1975). Stochastic Differential Equations and Applications, Vol. 1, New York, Academic Press, New York.

[32] Churchhouse, R. F. (Ed.) (1981). Handbook of Applicable Mathematics, Vol. III: Numerical Methods, Chichester, John Wiley \& Sons. 

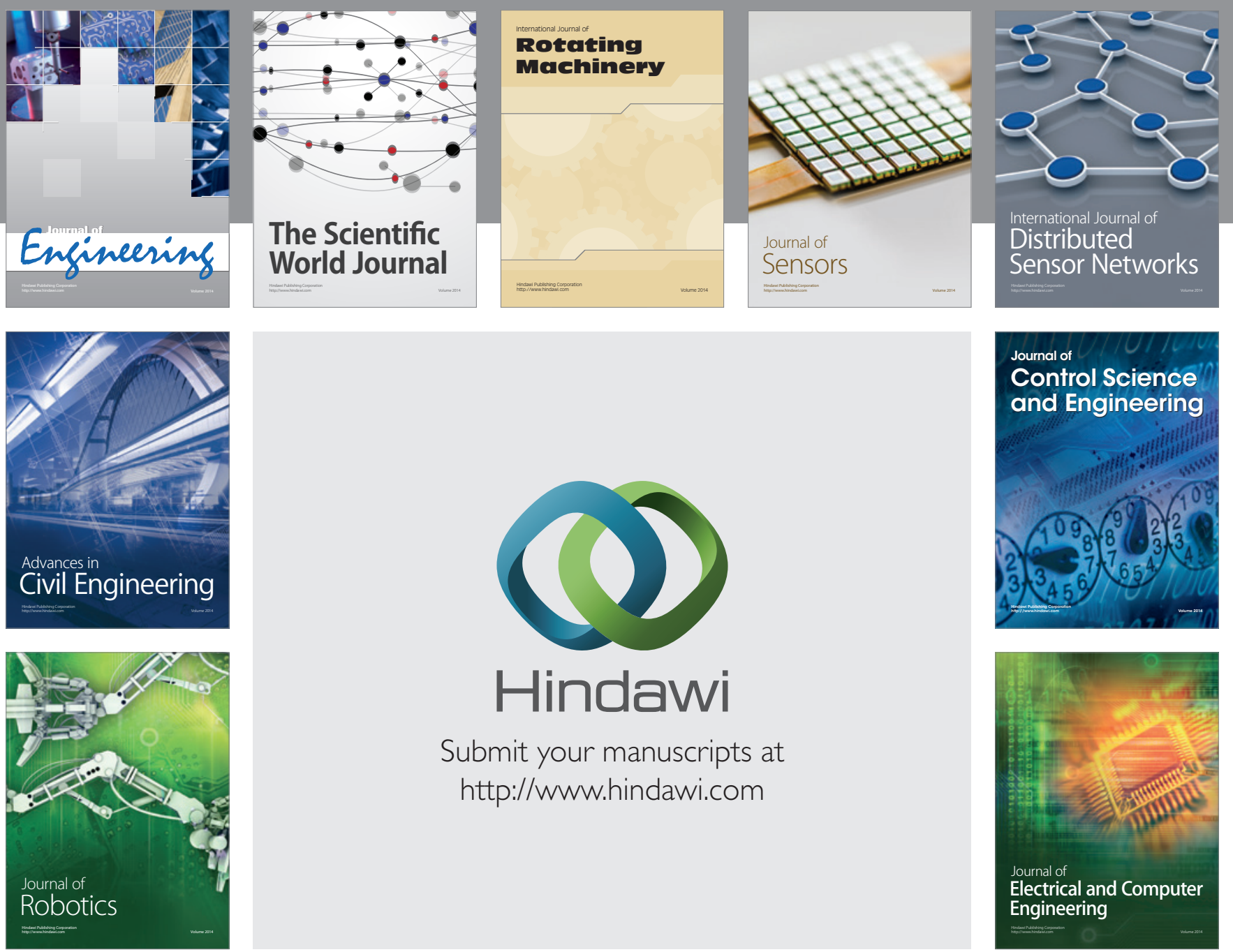

Submit your manuscripts at

http://www.hindawi.com
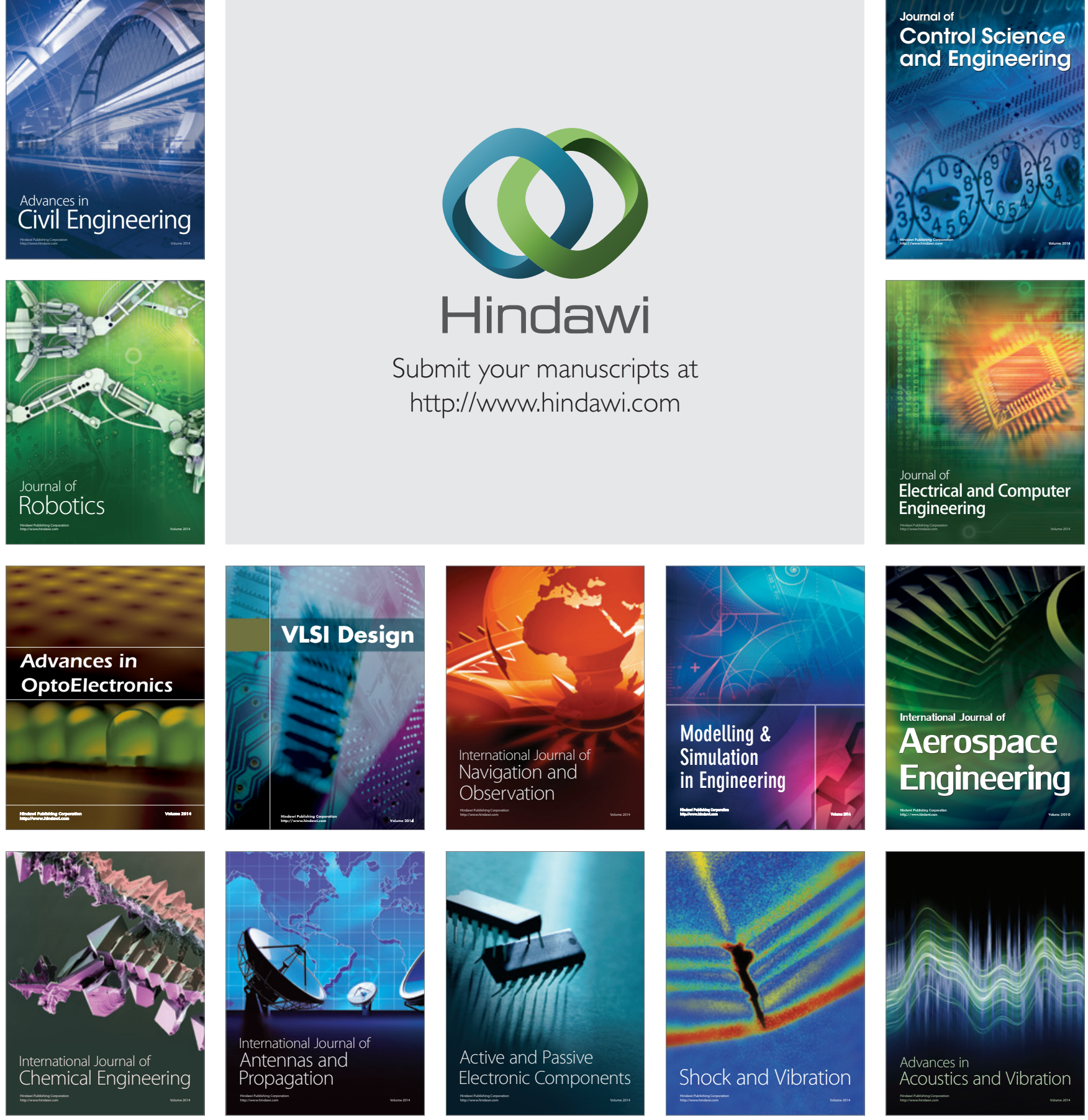